\title{
Evaluation of Cost-Effectiveness Criteria in Supply Chain Management: Case Study
}

\author{
Reza Rostamzadeh, ${ }^{1,2}$ Mahdi Sabaghi, ${ }^{3}$ and Ahmad Esmaili ${ }^{4}$ \\ ${ }^{1}$ Department of Management, Faculty of Management and Human Resource Development, Universiti Teknologi Malaysia, 81310 \\ Skudai, Johor, Malaysia \\ ${ }^{2}$ Department of Management, East Azarbaijan Science \& Research Branch, Islamic Azad University, Tabriz 51579-44533, Iran \\ ${ }^{3}$ Department of Mechanical Engineering, École Polytechnique de Montréal, C.P. 6079 Succ. Centre-Ville, Montréal, QC, Canada H3C \\ $3 A 7$ \\ ${ }^{4}$ Department of Industerial Management, Faculty of Accounting and Management, Allameh Tabataba'i University, Tehran 1434863111, \\ Iran
}

Correspondence should be addressed to Reza Rostamzadeh; rostamzadeh59@gmail.com

Received 28 June 2013; Revised 13 September 2013; Accepted 14 September 2013

Academic Editor: Wai Ki Ching

Copyright (C) 2013 Reza Rostamzadeh et al. This is an open access article distributed under the Creative Commons Attribution License, which permits unrestricted use, distribution, and reproduction in any medium, provided the original work is properly cited.

\begin{abstract}
The aim of this paper is to evaluate and prioritize the proposed cost-effectiveness criteria in supply chain management using fuzzy multiple attribute decision-making (MADM) approach. Over the past few years, the determination of suitable cost-effectiveness criteria in the supply chain has become a key strategic issue. However, the nature of these kinds of decisions is usually complex and unstructured. Many quantitative and qualitative factors must be considered to determine the suitable criteria. As the human decision-making process usually contains fuzziness and vagueness, a hierarchy of MADM model based on fuzzy-sets theory is used in this research. Using a fuzzy analytic hierarchy process (FAHP), the weights of criteria and subcriteria are determined and then the final ranking is determined by technique for order preference by similarity to ideal solution (TOPSIS). Finally, fuzzy TOPSIS (FTOPSIS) is employed to compare the results with classic TOPSIS. This paper concludes that the subcriteria in all the items are in the same rank.
\end{abstract}

\section{Introduction}

Supply chain plays a critical role for a company to gain competitive advantage, since the supply chain affects customer service, inventory and distribution costs, and response to the ever-changing markets directly. Furthermore, this role becomes more critical in today's distributed manufacturing environment in which companies focus on core competencies and outsource supportive tasks which in turn create large supply networks. In an international marketplace, an increasingly tough competition results in a company's attempt to find strategies which give them more competitive advantage than their rivals [1]. In fact, the competition is among supply chains, not companies [2]. Typically, supply chain inventory management studies are classified into three stages, which are supply, production, and distribution [3]; however, the main focus is usually placed on the coordination between only two of them.

The main objective of this research is to propose a systematic evaluation model which eases the way for manufacturing companies to select and find the most important costeffective criteria in supply chain management (SCM) under fuzzy multievaluator and multicriteria environment. Hence, this study utilizes multicriteria decision-making method to determine the importance weights of evaluation criteria in linguistic terms parameterized with triangular fuzzy numbers. This approach is employed for four reasons: (a) it is rational and understandable; (b) the computation processes are straightforward; (c) the concept permits the pursuit of the best alternatives for each criterion depicted in a simple mathematical form; and (d) the importance weights are incorporated into the comparison procedures $[4,5]$. 
The remainder of this paper is organized as follows. Section 2 presents the literature review of the supply chain modelling. In Section 3, methodologies of TOPSIS and FTOPSIS are given. An application of case study is given in Section 4. And finally in Section 5, the results of the application with two approaches (TOPSIS and FTOPSIS) are presented and suggestions for the future studies are clarified.

\section{The Literature Review}

An integrated supply chain multi-objective model was developed by Sabri and Beamon [6] and applied in strategic planning and operational SC. The objective of the strategic model was to minimize the costs of the chain, while the objective of operational level was to determine the amount of equipment purchased and distribution using the economic order quantity. A deterministic mixed integer, nonlinear mathematical programming model based on economic order quantity (EOQ) technique was offered by Cohen and Lee [7], which extends the overall policy of resource deployment. The total profit after tax for manufacturing facilities and distribution centers was maximized in the objective function. Also, management constraints, logical consistency constraints, and material requirements and assignments for all the products were considered. Petrovic et al. [8] describe fuzzy modeling and simulation of an SC in an uncertain environment. The goal was to find the inventory levels and order quantities for each inventory in an SC to ensure satisfactory performance at a reasonable total cost for the entire SC. Sources of uncertainty in customer's demand and external supply of raw materials have been taken into account and represented by fuzzy sets, and then a special SC simulator was developed. Currently, it does not consider the real scope of variables and constraints in a supply chain network (SCN). The PLANWAR model developed by Pirkul and Jayaraman [9] is a new formulation of multiproduct, multisite, problem capacitation location of the facility intended to address a number of plants and distribution centers so that the total operating costs for the distribution network were minimized. In fact, they have developed an MIP model for the plant and the problem of the warehouse location. In addition, Vidal and Goetschalck [10] modeled a Global Logistics System (GLS). By using historical data, the system approximates the probability that the supplier sends shipments on time, and the reliability of suppliers has been modeled. The effect of exchange rates, changes in demand, and international transit times were also investigated. Analytical models have been proposed by Lee and Kim [11] to solve the integrated production-distribution problems in supply chain management. They proposed an integrated multiperiod, multiproduct, and multishop production and distribution model in supply chain to gratify the retailer's demand in the given time periods. For the demand problem, they developed a hybrid method that combined the analysis and simulation model. Analytical model minimizes the overall costs of production, distribution, inventory holding, and shortage costs, subjected to various kinds of inventory and operation time constraints. Yin et al. [12] proposed a model for supply chain by taking into account both multi-input and multioutput data, based on the concept of virtual marketplace with multiagents. In addition, independent production and production support were made by the bidding strategies of demand/supply officers. The simulation experiment shows the applicability of economic analysis of this framework in a dynamic environment.

Nonino and Panizzolo [13] analyzed the problem of integrating production and distribution in a single place of supply and demand of several places with a focus on improving and streamlining the distribution network and then offered effective solutions rather than considering the routing problem at both strategic and operational perspectives. Ross and Jayaraman [14] extended the work by Jayaraman and Ross [15] and provided an assessment of the new heuristic solution procedures for the location of cross docks and distribution centers in the network design of SC. The authors described two heuristic solutions as close to the optimal design of distribution systems. Jeong et al. [16] proposed a mixed model of supply network design and planning of production/distribution. The authors used Lagrangian heuristic for the design of SCN and implemented genetic algorithm (GA) for the problem of integrated productiondistribution planning. However, their model also ignores the dynamic environment in which demand forecasts change over time, but the model was not well integrated because they proposed separate models for the three subnetworks. Yu et al. [17] examined the inventory period of several deterministic routing problems with a split delivery (IRPSD). All clients' requests were identified and met without being backordered. The model only describes the solution in each period when the quantity was delivered to each customer, carried by each directed arc and the number of times it was visited by vehicles in the transportation thereof.

Seliaman [18] developed a four-stage, serial supply chain inventory model with planned backorders. This chain consists of a supplier, a manufacturer, a distributor, and an end retailer. Production and inventory decisions are made at the supplier, manufacturer, and distributor levels. The production and demand rates were assumed as finite. In addition, backorders were permitted for demands that were not met at the retailer. The problem occurs in coordinating production and inventory decisions across the supply chain, so that the total cost of the system was minimized. Amirteimoori and Khoshandam [19] applied a DEA model for measuring the performance of suppliers and manufacturers in supply chain operations. Additive efficiency decomposition for suppliers and manufacturers in supply chain operations was proposed.

Based on the literature review for the concept of supply chain models, there are a few researches on the research topic and most of them have been conducted by focusing on production and distribution criteria. There is a close connection between the design and management of the supply chain flow (product, information, and funds) and the success of a supply chain which can be partly stated that many of e-businesses failures are due to the weaknesses in their supply chain design and planning. Regarding the literature review, this research proposes new cost-oriented criteria for evaluation in SCM using fuzzy MADM to help an organization to make unified and satisfactory decisions. 


\section{Methodology}

In this section, firstly TOPSIS method is summarized and then followed by summary of FTOPSIS method.

3.1. TOPSIS. Hwang and Yoon [20] originally proposed the order performance technique based on similarity to ideal solution (TOPSIS), in which the chosen alternative should not only have the shortest distance from the positive ideal reference point (PIRP), but also the longest distance from the negative ideal reference point (NIRP) to solve the multicriteria decision-making (MCDM) problems. Chen [21] extended TOPSIS method to the fuzzy environment. Wang and Elhag [22] suggested a fuzzy TOPSIS model, where ratings of alternatives under the criteria and importance weights of criteria are assessed in linguistic values represented by fuzzy numbers. Wang and Chang [23] applied the fuzzy MCDM method to determine the importance weights of evaluation criteria and to synthesize the ratings of candidate's aircraft, then TOPSIS is employed to obtain a crisp overall performance value for each alternative to make a final decision. Other studies can be found in Yang and Hung, [24]; Wang and Lee, [25]; Kahraman et al. [26]; Dağdeviren et al. [27]; Gumus, [28]; Sun and Lin, [29], Torfi and Rashidi, [30]; Armero et al. [31]. In this study, TOPSIS method is used for the final ranking of the cost-effectiveness criteria, and then the results are compared with fuzzy TOPSIS. The steps in TOPSIS are given as follows.

Step 1. Decision matrix is normalized via

$$
r_{i j}=\frac{w_{i j}}{\sqrt{\sum_{j=1}^{J} w_{i j}^{2}}}, \quad j=1,2,3, \ldots, J, i=1,2,3, \ldots, n .
$$

Step 2. The weighted normalized decision matrix is formed by

$$
v_{i j}=w_{i} * r_{i j}, \quad j=1,2,3, \ldots, J, i=1,2,3, \ldots, n .
$$

Step 3. Positive ideal solution (PIS) and negative ideal solution (NIS) will be determined by

$$
\begin{array}{ll}
A^{+}=\left\{v_{1}^{+}, v_{2}^{+}, v_{3}^{+}, \ldots, n\right\} & \text { max values, } \\
A^{-}=\left\{v_{1}^{-}, v_{2}^{-}, v_{3}^{-}, \ldots, n\right\} & \text { min values. }
\end{array}
$$

Step 4. The distance of each alternative from PIS and NIS is calculated as

$$
\begin{aligned}
& d_{i}^{+}=\sqrt{\sum_{j=1}^{n}\left(v_{i j}-v_{j}^{+}\right)^{2}}, \quad j=1,2, \ldots, J, \\
& d_{i}^{-}=\sqrt{\sum_{j=1}^{n}\left(v_{i j}-v_{j}^{-}\right)^{2}}, \quad j=1,2, \ldots, J .
\end{aligned}
$$

Step 5. The closeness coefficient of each alternative is calculated as

$$
\mathrm{CC}_{i}=\frac{d_{i}^{-}}{d_{i}^{+}+d_{i}^{-}}, \quad i=1,2, \ldots, J
$$

Step 6. By comparing $\mathrm{CC}_{i}$ values, the ranking of alternatives is determined.

3.2. Fuzzy TOPSIS. Here, the steps of fuzzy TOPSIS developed by Chen and Hwang [32] are given. First, a decision matrix $(D)$ of $m \times n$ dimension is defined as in

$$
\begin{gathered}
x_{1} \\
\\
D=A_{1} \\
A_{i} \\
A_{m}
\end{gathered}\left(\begin{array}{ccccc}
\tilde{x}_{11} & \ldots & \tilde{x}_{1 j} & \ldots & \tilde{x}_{1 n} \\
\tilde{x}_{i 1} & \ldots & \tilde{x}_{i j} & \ldots & \tilde{x}_{i n} \\
\tilde{x}_{m 1} & \ldots & \tilde{x}_{m j} & \ldots & \tilde{x}_{m n}
\end{array}\right),
$$

where $x_{i j}, \forall_{i j}$ may be crisp or fuzzy. If $x_{i j}$ is fuzzy, it is represented by a triangular fuzzy number (TFN) as $\tilde{x}_{i j}=$ $\left(a_{i j}, b_{i j}, c_{i j}\right)$. The fuzzy weights can be described by

$$
W=\left(\widetilde{w}_{1}, \ldots, \widetilde{w}_{j}, \ldots, \widetilde{w}_{n}\right) ; \quad \widetilde{w}_{j}=\left(\alpha_{j}, \beta_{j}, \chi_{j}\right) .
$$

The problem is solved using the following steps.

Step 1. Normalize the decision matrix. The decision matrix must be first normalized so that the elements are unit-free. A linear normalization method was used as

$$
\tilde{r}_{i j}= \begin{cases}\tilde{x}_{i j}(/) \tilde{x}_{j}^{+}=\left(\frac{a_{i j}}{c_{j}^{+}}, \frac{b_{i j}}{b_{j}^{+}}, \frac{c_{i j}}{a_{j}^{+}}\right) & \forall_{j}, x_{j} \text { is a benefit attribute } \\ \tilde{x}_{j}^{-}(/) \tilde{x}_{i j}=\left(\frac{a_{j}^{-}}{c_{i j}}, \frac{b_{j}^{-}}{b_{i j}}, \frac{c_{j}^{-}}{a_{i j}}\right) & \forall_{j}, x_{j} \text { is a cost attribute. }\end{cases}
$$

By applying (3), we can rewrite the decision matrix in (6) as in

$$
\begin{aligned}
& \begin{array}{lllll}
x_{1} & \ldots & x_{j} & \ldots & x_{n}
\end{array} \\
& D^{\prime}=\begin{array}{c}
A_{1} \\
A_{i} \\
A_{m}
\end{array}\left(\begin{array}{ccccc}
\tilde{r}_{11} & \ldots & \tilde{r}_{1 j} & \ldots & \tilde{r}_{1 n} \\
\tilde{r}_{i 1} & \ldots & \tilde{r}_{i j} & \ldots & \tilde{r}_{i n} \\
\tilde{r}_{m 1} & \ldots & \tilde{r}_{m j} & \ldots & \tilde{r}_{m n}
\end{array}\right) \text {. }
\end{aligned}
$$

Step 2. Calculate the weighted normalized fuzzy decision matrix. The weighted normalized value $\widetilde{v}_{i j}$ calculated by

$$
\begin{aligned}
& \widetilde{v}_{i j}=\widetilde{r}_{i j}(\cdot) \widetilde{w}_{j}^{+}=\left(\frac{a_{i j}}{c_{j}^{*}} \alpha_{j}, \frac{b_{i j}}{b_{j}^{*}} \beta_{j}, \frac{c_{i j}}{a_{j}^{*}} \chi_{j}\right), \\
& \widetilde{v}_{i j}=\widetilde{r}_{i j}(\cdot) \widetilde{w}_{j}=\left(\frac{a_{j}^{-}}{c_{i j}} \alpha_{j}, \frac{b_{j}^{-}}{b_{i j}} \beta_{j}, \frac{c_{j}^{-}}{a_{i j}} \chi_{j}\right) .
\end{aligned}
$$

Equation (10) is used when the $j$ th attribute is a benefit attribute. Equation (11) is used when the $j$ th attribute is a cost attribute. The result can be summarized as in

$$
\begin{aligned}
& X_{1} \quad X_{j} \quad X_{n} \\
& v=\begin{array}{c}
A_{1} \\
A_{i} \\
A_{m}
\end{array}\left(\begin{array}{ccccc}
v_{11} & \ldots & v_{1 j} & \ldots & v_{1 n} \\
v_{i 1} & \ldots & v_{i j} & \ldots & v_{i n} \\
v_{m 1} & \ldots & v_{m j} & \ldots & v_{m n}
\end{array}\right) .
\end{aligned}
$$


Step 3. Identify (PIS), $\left(A^{+}\right)$, and (NIS), $\left(A^{-}\right)$solutions.

$v_{j}^{+}$and $v_{j}^{-}$may be obtained through some ranking procedures. Chen and Hwang [32] used Lee and Li's ranking method for comparison of fuzzy numbers. The $v_{j}^{+}$and $v_{j}^{-}$are the fuzzy numbers with the largest and the smallest generalized mean, respectively. The generalized mean for fuzzy number, $v_{i j}, \forall_{j}, j$, is defined as

$$
M\left(\widetilde{v}_{i j}\right)=\frac{-a_{i j}^{2}+c_{i j}^{2}-a_{i j} \cdot b_{i j}+c_{i j} \cdot b_{i j}}{3\left(-a_{i j}+c_{i j}\right)} .
$$

For each column $j$, we find a $v_{i j}$ whose greatest mean is $v_{j}^{+}$and lowest mean is $v_{j}^{-}$.

Step 4. Calculate the distance of each alternative from $A^{+}$ and $A^{-}$. For fuzzy data, the difference between two fuzzy numbers is explained as given in

$$
\begin{aligned}
& D_{i j}^{+}=1-\sup _{x}\left\{\min \left[\propto_{v_{i j}}(x), \propto_{v_{j}^{+}}(x)\right]\right\}, \\
& D_{i j}^{-}=1-\sup _{x}\left\{\min \left[\propto_{v_{i j}}(x), \propto_{v_{j}^{-}}(x)\right]\right\},
\end{aligned}
$$

in which this equation is extendable for FTNs as follows.

$$
\text { If } v_{j}^{+}=\left(a^{+}, b^{+}, c^{+}\right) \text {and } v_{j}^{-}=\left(a^{-}, b^{-}, c^{-}\right) \text {, then }
$$

$$
\begin{gathered}
D_{i j}^{+}= \begin{cases}1-\frac{c_{i j}-a^{+}}{b^{+}+c_{i j}-a^{+}-b_{i j}} & \text { for }\left(b_{i j}<b^{+}\right), \\
1-\frac{c^{+}-a_{i j}}{b_{i j}+c^{+}-a_{i j}-b^{+}} & \text {for }\left(b^{+}<b_{i j}\right),\end{cases} \\
D_{i j}^{-}= \begin{cases}1-\frac{c^{-}-a_{i j}}{b_{i j}+c^{-}-a_{i j}-b^{-}} & \text {for }\left(b^{-}<b_{i j}\right), \\
1-\frac{c_{i j}-a^{-}}{b^{-}+c_{i j}-a^{-}-b_{i j}} & \text { for }\left(b_{i j}<b^{-}\right) .\end{cases}
\end{gathered}
$$

Note that both $D_{i j}^{+}$and $D_{i j}^{-}$are crisp numbers.

Step 5. Compute the relative closeness to ideals. This index is used to combine $S_{i}^{+}$and $S_{i}^{-}$indices calculated in Step 4. Since $S_{i}^{+}$and $S_{i}^{-}$are crisp numbers, they can be combined

$$
\mathrm{CC}_{i}=\frac{S_{i}^{-}}{S_{i}^{+}+S_{i}^{-}} \quad i=1,2, \ldots, I .
$$

Step 6. Rank preference order. Choose an alternative with maximum $\mathrm{CC}_{i}^{+}$or rank alternatives according to $\mathrm{CC}_{i}^{-}$in descending order.

\section{Case Study}

SCM has influenced human lives, thoughts, and actions for more than a decade. Practitioners and academicians have spent several years in comprehending and exploring the complexity of SCM. The evolution has been slow but stable from logistic to material management to SCM. The 1990s have been the stormiest period, leading to large-scale acceptance of SCM concept. The change in power from the manufacturer to the consumer, accessibility and technology, the arrival of the omnipresent Internet, and economic deregulation leading to firm's competition are just some of the characteristics of this new age. This in turn prompted change from the obligation of making profit and wealth from the market (external environment outside the manufacturer's control) to the organization itself (within the manufacturer's control). The tools and techniques of SCM have appeared to be the manufacturer's lifeline. The benefits of SCM are multiples and long term [33].

Company $X$ is an Iranian firm which is active in the toy industries. Toy business is tremendously unstable and seasonal in nature, which is relatively different from the industries like chemical, telecommunication, agriculture, pharmaceutical, automobile, and so on [34]. The volatility in the toy industry is caused by variables and disorganized demands, very short and specific selling-windows, and short product-life cycles. Hence, investors and practitioners know very well that the toy industry is far from tranquil [35]. Compared to other industries, toy industry has suffered comparatively higher costs on outdated inventory, lost sales, and markdown. These are the distinctive consequences of volatility in the toy supply chains, similar to the fashion clothing industry [36]. In facing a very unique challenge, these industries need to survive by providing the right toys at the right quantity and at the right stores during very short selling windows, and also to frequently provide creative and yet price-competitive toys.

Over the past few years, the determination of suitable cost-effectiveness criteria in the supply chain has become a key strategic issue. However, the nature of these decisions is usually complex and unstructured. In general, many quantitative and qualitative factors must be considered to determine suitable criteria. The managers and analysts of the company decided to evaluate their supply chain situation to make strategic decisions for the future and gain a strong business relationship with the suppliers. The most important cost-effectiveness criteria from three areas including supply, production, and distribution sections have been collected through the interview with the managers and experts of the company. To determine the reliability of the questionnaire, test-retest method was used. In this way, the researcher randomly chose five samples from managers at two different times (at least two weeks), then questionnaires were distributed to them. After that, Spearman rank correlation coefficient analysis and meaningful test of sampling were conducted. Reliability test of the questionnaire was conducted at $97 \%$ confidence coefficient level. As the results show, the questionnaire has acceptable reliability. After determining the criteria and subcriteria, decision tree of the problem was designed. Figure 1 shows the hierarchical process of cost criteria.

There is a main goal in the first level, which is an evaluation of the cost-effectiveness criteria in supply chain, 3 criteria at the second level, and at the third level, 20 subcriteria have been located in 3 different groups. The aim of this research is the evaluation and prioritization of proposed 


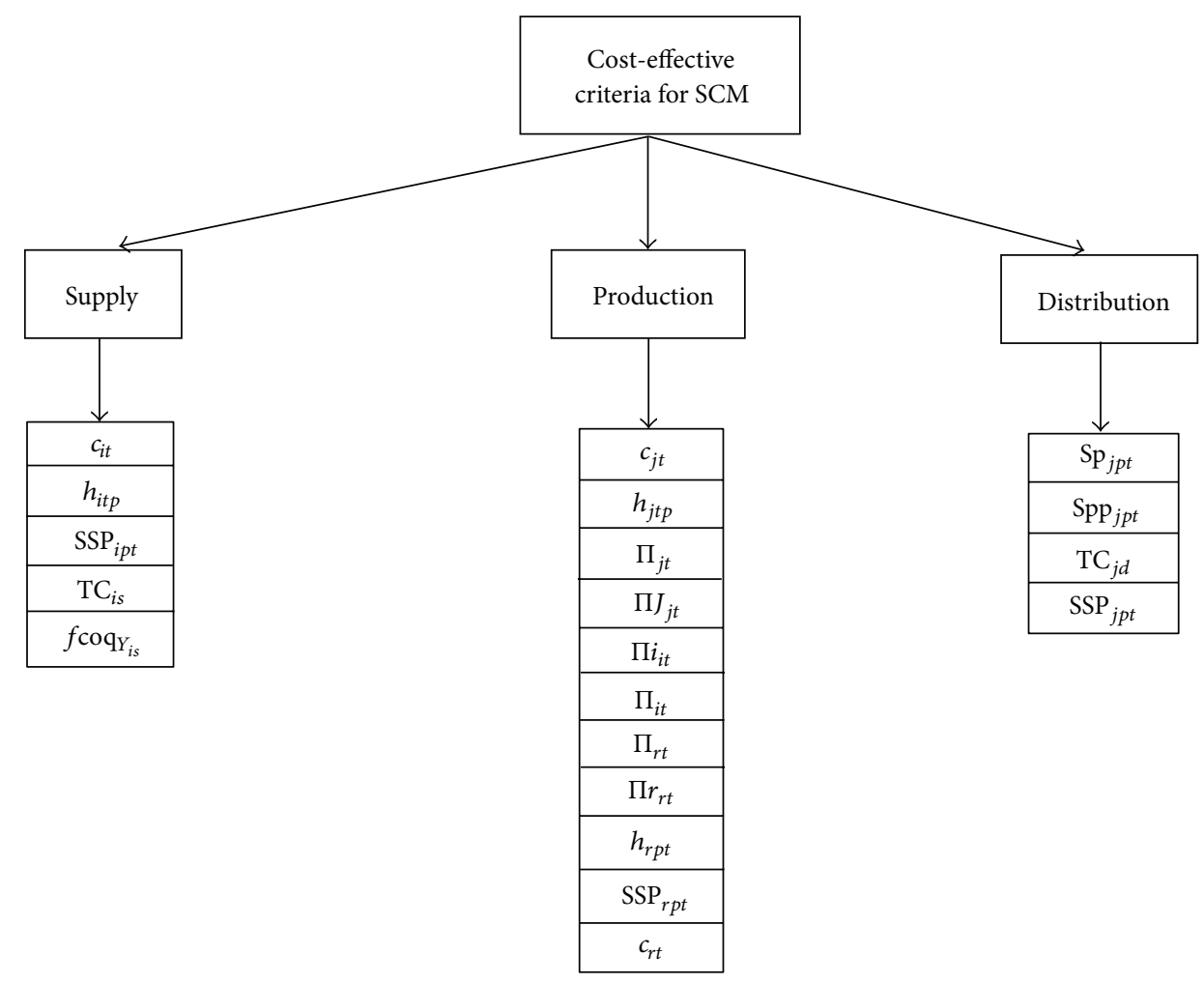

FIGURE 1: Hierarchal process for the criteria.

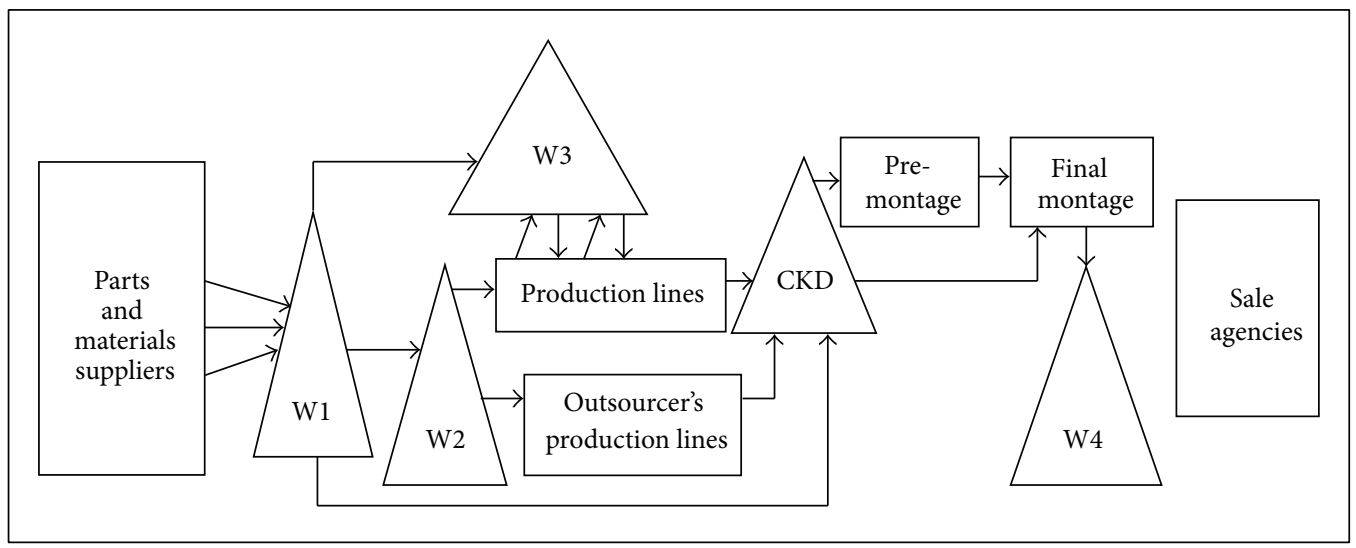

FIGURE 2: Supply chain of the company.

cost-effectiveness criteria in supply chain management. This research has been done in three stages. In the first stage, to determine the weights of criteria, FAHP was used, and for the final ranking of the same criteria, TOPSIS was applied. Then, fuzzy TOPSIS was applied to compare the results with the classic TOPSIS. The supply chain of the company is shown in Figure 2.

The raw materials of parts are obtained through four ways, which are domestic suppliers, international suppliers, internal production, and outsourcing. All materials are held in warehouse (W1) at the entry point and then the materials are separated accordingly. Raw materials are stored in warehousing (W2) while semimanufactured parts are stored in warehouses (W3). The parts which are made inside the factory and the parts that are made by the outsourcers are transferred to the Complete Knock Down (CKD) warehouse. The parts are then transferred from CKD to the montage and premontage workshops. The finished products are transported to the finished products warehouse (W4), and after that they are sent to the sales agencies for distribution to the customers. Regarding the above description, the indices used in this research are defined as Indices Section.

4.1. Application of FAHP. The respondents of this research were managers, assistant managers, analysts, and experts of the company (which is a manufacturing firm). Questionnaire 
TABLE 1: Linguistic scale of importance.

\begin{tabular}{lcc}
\hline $\begin{array}{l}\text { Linguistic scale for } \\
\text { importance }\end{array}$ & $\begin{array}{c}\text { Triangular fuzzy } \\
\text { scale }\end{array}$ & $\begin{array}{c}\text { Triangular fuzzy } \\
\text { reciprocal scale }\end{array}$ \\
\hline Equal & $(1,1,1)$ & $(1,1,1)$ \\
Weak & $(1 / 2,1,3 / 2)$ & $(2 / 3,1,2)$ \\
Fairly strong & $(3 / 2,2,5 / 2)$ & $(2 / 5,1 / 2,2 / 3)$ \\
Very strong & $(5 / 2,3,7 / 2)$ & $(2 / 7,1 / 3,2 / 5)$ \\
Absolute & $(7 / 2,4,9 / 2)$ & $(2 / 9,1 / 4,2 / 7)$ \\
\hline
\end{tabular}

was used to gather the data needed for FAHP tables. In this research, we have asked for managers' and experts' opinions through questionnaires to rank the cost-effectiveness criteria in SCM. Table 1 shows the linguistic scales of the important criteria. Then, the criteria were calculated using FAHP. Calculations of subcriteria were done in the same manner. In Table 2, the fuzzy evaluation matrix sample for main criteria of supply is given.

After the normalization of these values, priority weights with respect to main goal were calculated as $W=$ $\left(\begin{array}{ccc}\stackrel{S}{S} & P & D \\ 0.475 & 0.43 & 0.095\end{array}\right)$. Then, weights of subcriteria were calculated similarly. Final weights of criteria and subcriteria using FAHP are given in Table 3. These weights will be used in TOPSIS process.

4.2. Application of TOPSIS. In this stage, TOPSIS is used to obtain the final ranks of criteria. For this reason, we have grouped the managers and analysts of the company into 3 groups of decision makers. Decision makers from different backgrounds may define different weight vectors. They usually cause not only imprecise evaluation, but also serious persecution during the decision process [37]. The linguistic evaluation is shown in Table 4 . Also, Table 5 represents the importance weights of criteria from three decision makers. Then, the normalized-decision matrix and weighted normalized-decision matrix were constructed. Table 6 shows the final weights of criteria and subcriteria using TOPSIS.

After normalizing via (1), $R$ will be obtained as follows:

$$
R=\left[\begin{array}{lll}
0.672 & 0.703 & 0.329 \\
0.523 & 0.502 & 0.768 \\
0.523 & 0.502 & 0.548
\end{array}\right] .
$$

The weights that we obtained from FAHP were used to get the weighted decision matrix $V$. So, weighted normalized decision matrix was formed by (2):

$$
V=\left[\begin{array}{lll}
0.317 & 0.302 & 0.031 \\
0.248 & 0.215 & 0.072 \\
0.248 & 0.215 & 0.052
\end{array}\right] .
$$

Then positive ideal solution (PIS) and negative ideal solution (NIS) will be determined by (3):

$$
\begin{aligned}
& A^{+}=\{0.317,0.302,0.072\}, \\
& A^{-}=\{0.248,0.215,0.031\} .
\end{aligned}
$$

TABLE 2: The fuzzy evaluation matrix with respect to the goal.

\begin{tabular}{lccc}
\hline $\begin{array}{l}\text { Cost-effectiveness } \\
\text { criteria }\end{array}$ & Supply & Production & Distribution \\
\hline Supply & $(1,1,1)$ & $(1 / 2,1,3 / 2)$ & $(3 / 2,2,5 / 2)$ \\
Production & $(2 / 3,1,2)$ & $(1,1,1)$ & $(3 / 2,2,5 / 2)$ \\
Distribution & $(2 / 5,1 / 2,2 / 3)$ & $(2 / 5,1 / 2,2 / 3)$ & $(1,1,1)$ \\
\hline
\end{tabular}

TABLE 3: Final weights of criteria and subcriteria using FAHP.

\begin{tabular}{lccc}
\hline Rank & Supply $(0.475)$ & Production (0.43) & Distribution (0.095) \\
\hline 1 & $c_{i t}(0.331)$ & $\prod i_{i t}(0.154)$ & $\operatorname{Spp}_{j p t}(0.409)$ \\
2 & $f \operatorname{coq}_{Y_{i s}}(0.312)$ & $c_{j t}(0.147)$ & $\mathrm{TC}_{j d}(0.298)$ \\
3 & $h_{i t p}(0.173)$ & $\prod_{i t}(0.14)$ & $\operatorname{Sp}_{j p t}(0.231)$ \\
4 & $\operatorname{SSP}_{i p t}(0.126)$ & $c_{r t}(0.11)$ & $\operatorname{SSP}_{j p t}(0.06)$ \\
5 & $\operatorname{TC}_{i s}(0.55)$ & $\prod_{j t}(0.098)$ & \\
6 & & $\prod j_{j t}(0.087)$ & \\
7 & & $\prod r_{r t}(0.074)$ & \\
8 & & $h_{r p t}(0.057)$ & \\
9 & & $\prod_{r t}(0.046)$ & \\
10 & & $\operatorname{SSP}_{r p t}(0.043)$ & \\
11 & & $h_{j t p}(0.035)$ & \\
\hline
\end{tabular}

The distance of each alternative from PIS and NIS was calculated through (4):

$$
\begin{array}{lll}
d_{1}^{+}=0.041, & d_{2}^{+}=0.111, & d_{3}^{+}=0.112, \\
d_{1}^{-}=0.111, & d_{2}^{-}=0.041, & d_{3}^{-}=0.021 .
\end{array}
$$

The closeness coefficients of each alternative were calculated by (5):

$$
\begin{gathered}
C_{1}^{+}=\frac{d_{1}^{-}}{d_{1}^{+}+d_{1}^{-}}=\frac{0.111}{0.041+0.111}=0.73, \\
C_{2}^{+}=\frac{0.041}{0.111+0.041}=0.269, \\
C_{3}^{+}=\frac{0.021}{0.112+0.021}=0.157 .
\end{gathered}
$$

Comparing $\mathrm{CC}_{i}$ values, the ranking of main criteria was determined as follows:

$$
C_{1}>C_{2}>C_{3} .
$$

So we can arrange the final weight of the main criteria as Table 6.

4.3. Application of FTOPSIS. In the third stage, FTOPSIS was implemented to compare the results with classic TOPSIS. The linguistic evaluations are shown in Tables 7 and 8. Meanwhile, Table 9 illustrates the importance weight of criteria from three groups of decision makers. Tables 7 and 8 were converted into triangular fuzzy numbers to construct the fuzzy-decision matrix and determine the fuzzy weight of each 
TABLE 4: Linguistic variables for the criteria weights.

\begin{tabular}{ll}
\hline Very low $(\mathrm{VL})$ & 1 \\
Low $(\mathrm{L})$ & 3 \\
Medium $(\mathrm{M})$ & 5 \\
High $(\mathrm{H})$ & 7 \\
Very high $(\mathrm{VH})$ & 9 \\
\hline
\end{tabular}

TABLE 5: Importance weight of criteria from three decision makers for main criteria.

\begin{tabular}{lccc}
\hline Criteria & Supply & Production & Distribution \\
\hline Decision makers & & & \\
$D_{1}$ & 9 & 7 & 3 \\
$D_{2}$ & 7 & 5 & 7 \\
$D_{3}$ & 7 & 5 & 5 \\
\hline$W$ & 0.475 & 0.43 & 0.095
\end{tabular}

criterion, as shown in Table 10. Then, the normalized fuzzydecision matrix and weighted normalized fuzzy-decision matrix were constructed.

Step 1. Normalize the decision matrix:

$\begin{array}{cccc} & \text { Supply } & \text { Production } & \text { Distribution } \\ \tilde{x}_{j}^{+} & (0.8,1,1) & (0.7,0.8,0.9) & (0.6,0.7,0.8) \\ \tilde{x}_{j}^{-} & (0.7,0.8,0.9) & (0.6,0.7,0.8) & (0.35,0.5,0.65)\end{array}$

$\tilde{r}_{11}=\tilde{x}_{11}(/) \tilde{x}_{1}^{+}=\left(\frac{0.7}{1}, \frac{0.8}{1}, \frac{0.9}{0.8}\right)=(0.7,0.8,1.125)$

Therefore, the normalized matrix is

$$
\begin{array}{ccc}
x_{1} & x_{2} & x_{3} \\
D^{\prime}=A_{1} & & \\
A_{2} \\
A_{3}
\end{array}\left(\begin{array}{ccc}
(0.7,0.8,1.125) & (0.67,0.87,1.14) & (0.75,1,1.33) \\
(0.8,1,1.25) & (0.78,1,1.28) & (0.43,0.71,1.08) \\
(0.7,0.8,1.125) & (0.78,1,1.28) & (0.75,1,1.33)
\end{array}\right) .
$$

Step 2. Calculate the weighted normalized fuzzy decision matrix:

$$
\begin{aligned}
\widetilde{v}_{11}= & \widetilde{r}_{11}(\cdot) \widetilde{w}_{11}^{+}=(0.7,0.8,1.125) \\
& \times(0.8,1,1)=(0.56,0.8,1.125)
\end{aligned}
$$

Therefore the weighted normalized matrix is as follows:

$$
\begin{array}{ccc}
x_{1} & x_{2} & x_{3} \\
V=\begin{array}{c}
A_{1} \\
A_{2} \\
A_{3}
\end{array}\left(\begin{array}{ccc}
(0.56,0.8,1.125) & (0.469,0.696,1.026) & (0.45,0.7,1.064) \\
(0.64,1,1.25) & (0.546,0.8,1.15) & (0.258,0.497,0.864) \\
(0.56,0.8,1.125) & (0.546,0.8,1.15) & (0.45,0.7,1.064)
\end{array}\right) .
\end{array}
$$

TABLE 6: Final weights of criteria and subcriteria using TOPSIS.

\begin{tabular}{lccc}
\hline Rank & Supply (0.73) & Production $(0.269)$ & Distribution $(0.157)$ \\
\hline 1 & $f \operatorname{coq}_{Y_{i s}}(0.209)$ & $\prod i_{i t}(0.098)$ & $\operatorname{Spp}_{j p t}(0.253)$ \\
2 & $c_{i t}(0.204)$ & $c_{j t}(0.095)$ & $\mathrm{TC}_{j d}(0.215)$ \\
3 & $h_{i t p}(0.109)$ & $\prod_{i t}(0.08)$ & $\operatorname{Sp}_{j p t}(0.047)$ \\
4 & $\operatorname{SSP}_{i p t}(0.069)$ & $c_{r t}(0.063)$ & $\operatorname{SSP}_{j p t}(0.039)$ \\
5 & $\mathrm{TC}_{i s}(0.021)$ & $\prod_{j t}(0.0618)$ & \\
6 & & $\prod j_{j t}(0.0612)$ & \\
7 & & $\prod r_{r t}(0.037)$ & \\
8 & & $\prod_{r t}(0.035)$ & \\
9 & & $h_{r p t}(0.032)$ & \\
10 & & $\operatorname{SSP}_{r p t}(0.032)$ & \\
11 & & $h_{j t p}(0.015)$ & \\
\hline
\end{tabular}

TABLE 7: Linguistic variables for the criteria weights.

\begin{tabular}{lc}
\hline Very low $(\mathrm{VL})$ & $(0,0,0.2)$ \\
Low $(\mathrm{L})$ & $(0.1,0.2,0.3)$ \\
Medium low $(\mathrm{ML})$ & $(0.2,0.3,0.4)$ \\
Medium $(\mathrm{M})$ & $(0.35,0.5,0.65)$ \\
Medium high $(\mathrm{MH})$ & $(0.6,0.7,0.8)$ \\
High $(\mathrm{H})$ & $(0.7,0.8,0.9)$ \\
Very high $(\mathrm{VH})$ & $(0.8,1,1)$ \\
\hline
\end{tabular}

TABLE 8: Linguistic variables for the ratings.

\begin{tabular}{lc}
\hline Very poor $(\mathrm{VP})$ & $(0,0,0.2)$ \\
Poor $(\mathrm{P})$ & $(0.1,0.2,0.3)$ \\
Medium poor $(\mathrm{MP})$ & $(0.2,0.3,0.4)$ \\
Fair $(\mathrm{F})$ & $(0.35,0.5,0.65)$ \\
Medium good $(\mathrm{MG})$ & $(0.6,0.7,0.8)$ \\
Good $(\mathrm{G})$ & $(0.7,0.8,0.9)$ \\
Very good $(\mathrm{VG})$ & $(0.8,1,1)$ \\
\hline
\end{tabular}

TABLE 9: Importance weight of criteria from three decision makers.

\begin{tabular}{lccc}
\hline Criteria & Supply & Production & Distribution \\
\hline Decision makers & & & \\
$D_{1}$ & $\mathrm{H}$ & $\mathrm{MH}$ & $\mathrm{ML}$ \\
$D_{2}$ & $\mathrm{VH}$ & $\mathrm{H}$ & $\mathrm{M}$ \\
$D_{3}$ & $\mathrm{H}$ & $\mathrm{H}$ & $\mathrm{MH}$ \\
\hline
\end{tabular}

TABLE 10: Fuzzy-decision matrix and fuzzy weights for main criteria.

\begin{tabular}{lccc}
\hline Criteria & Supply & Production & Distribution \\
\hline$D_{1}$ & $(0.7,0.8,0.9)$ & $(0.6,0.7,0.8)$ & $\mathbf{( 0 . 6 , 0 . 7 , 0 . 8 )}$ \\
$D_{2}$ & $(\mathbf{0 . 8}, \mathbf{1}, \mathbf{1})$ & $(\mathbf{0 . 7}, \mathbf{0 . 8}, \mathbf{0 . 9})$ & $(0.35,0.5,0.65)$ \\
$D_{3}$ & $(0.7,0.8,0.9)$ & $(0.7,0.8,0.9)$ & $\mathbf{( 0 . 6 , 0 . 7 , 0 . 8 )}$ \\
\hline$W$ & $(0.8,1,1)$ & $(0.7,0.8,0.9)$ & $(0.6,0.7,0.8)$ \\
\hline
\end{tabular}

Bold font shows the Max. value of matrix and the Italic one shows the Min. value of matrix. 
Step 3. Identify positive ideal solution (PIS), $\left(A^{+}\right)$, and negative ideal solution (NIS), $\left(A^{-}\right)$solutions:

$$
\begin{aligned}
M\left(\widetilde{v}_{11}\right)= & \frac{(-0.56 \times 0.8)-0.56^{2}+1.25^{2}+(1.25 \times 0.8)}{3(1.25-0.56)} \\
= & 0.828 \\
& \vdots \\
M= & \left|\begin{array}{ccc}
0.828 & 0.73 & 0.73 \\
0.963 & 0.832 & 0.539 \\
0.828 & 0.832 & 0.73
\end{array}\right| \\
v_{1}^{+} & v_{2}^{+} \\
A^{+}=(0.64,1,1.25),(0.546,0.8,1.15),(0.45,0.7,1.064) & v_{3}^{+} \\
v_{1}^{-} & v_{2}^{-} \\
A^{-}= & (0.56,0.8,1.125),(0.469,0.696,1.026),(0.258,0.497,0.864) .
\end{aligned}
$$

Step 4. Calculate the distance of each alternative from $A^{+}$ and $A^{-}$

$$
\begin{aligned}
& D_{11}^{+}=1-\frac{1.125-0.64}{1+1.125-0.64-0.8}=0.291 \\
& D^{+}=\left(\begin{array}{ccc}
0.291 & 0 & 0 \\
0.291 & 0.178 & 0.329 \\
0 & 0 & 0
\end{array}\right) \\
& D_{33}^{-}=1-\frac{0.864-0.45}{0.7+0.864-0.45-0.497} \\
& =0.329 \\
& D^{-}=\left(\begin{array}{ccc}
0 & 0 & 0.329 \\
0 & 0.178 & 0 \\
0.291 & 0.178 & 0.329
\end{array}\right) \\
& S_{1}^{+}=0.291+0+0=0.291 \\
& S_{2}^{+}=0.291+0.178+0.329=0.798 \\
& S_{3}^{+}=0+0+0=0.341 \\
& S_{1}^{-}=0+0+0.329=0.329 \\
& S_{2}^{-}=0+0.178+0=0.178 \\
& S_{3}^{-}=0.291+0.178+0.329=0.798 \text {. }
\end{aligned}
$$

TABLE 11: Final rank of criteria and subcriteria using FTOPSIS.

\begin{tabular}{lccc}
\hline Rank & Supply (0.53) & Production (0.182) & Distribution $(0.7)$ \\
\hline 1 & $f \operatorname{Coq}_{Y_{i s}}(0.87)$ & $\prod i_{i t}(0.963)$ & $\operatorname{Spp}_{j p t}(0.73)$ \\
2 & $c_{i t}(0.832)$ & $c_{j t}(0.832)$ & $\mathrm{TC}_{j d}(0.66)$ \\
3 & $h_{i t p}(0.402)$ & $\prod_{i t}(0.75)$ & $\operatorname{Sp}_{j p t}(0.53)$ \\
4 & $\operatorname{SSP}_{i p t}(0.385)$ & $c_{r t}(0.7)$ & $\operatorname{SSP}_{j p t}(0.4)$ \\
5 & $\mathrm{TC}_{i s}(0.155)$ & $\prod_{j t}(0.685)$ & \\
6 & & $\prod j_{j t}(0.66)$ & \\
7 & & $\prod r_{r t}(0.593)$ & \\
8 & & $h_{r p t}(0.402)$ & \\
9 & & $\prod_{r t}(0.34)$ & \\
10 & & $\operatorname{SSP}_{r p t}(0.285)$ & \\
11 & & $h_{j t p}(0.244)$ & \\
\hline
\end{tabular}

Step 5. Compute the relative closeness to ideals:

$$
\begin{aligned}
& C_{1}=\frac{S_{1}^{-}}{S_{1}^{+}+S_{1}^{-}}=\frac{0.329}{0.291+0.329}=0.53, \\
& C_{2}=\frac{S_{2}^{-}}{S_{2}^{+}+S_{2}^{-}}=\frac{0.178}{0.798+0.178}=0.182, \\
& C_{3}=\frac{S_{3}^{-}}{S_{3}^{+}+S_{3}^{-}}=\frac{0.798}{0.341+0.798}=0.7, \\
& C_{3}>C_{1}>C_{2} .
\end{aligned}
$$

So, we can arrange the criteria as follows:

Distribution (0.7) > Supply (0.53) > Production (0.182).

Table 11 shows the final rank of FTOPSIS. Also the results from different methods were summarized in Table 12.

\section{Conclusions}

This paper applied approaches based on FAHP, TOPSIS, and FTOPSIS methods to evaluate and prioritize the costeffectiveness criteria in supply chain management. FAHP method was used to determine the weights of criteria and subcriteria, while TOPSIS method was applied for determination of the final ranking. Then, fuzzy TOPSIS was performed to compare the results with the classic TOPSIS. Both methods in this research resulted in the same rank. At the first level, there is a main goal to evaluate the cost-effectiveness criteria in supply chain, any 3 criteria at the second level and 20 subcriteria have been located in 3 groups at the third level. The model presented in this study is expected to enable the company to make satisfying and unified decisions in supply chain and also increases its competitive capabilities.

Consequently, after considering the research process, we can point out the findings from these items.

(1) By taking into account the meaning and concept of each criterion which demonstrates the situation of the organizational unit (manufacturing or service), the most important weight has been allocated to supply 
(TOPSIS: 0.73, FTOPSIS: 0.53 ). This is followed by production (TOPSIS: 0.269, FTOPSIS: 0.182) and distribution (TOPSIS: 0.159, FTOPSIS: 0.7). In most of the manufacturing companies, suppliers play an important role. The company must ensure about the availability of the materials and that the materials are provided in the right time, right place, and right amount. The only difference that can be observed is the distribution that has the highest score in FTOPSIS but the lowest score in TOPSIS.

(2) In supply groups, the most important weight has been allocated to the total cost of quality $\left(f \operatorname{coq}_{Y_{i s}}\right)$, (TOPSIS: 0.209, FTOPSIS: 0.87), and the lowest important weight is the transportation cost $\left(\mathrm{TC}_{i s}\right.$ ), (TOPSIS: 0.021, FTOPSIS: 0.155 ). The company has a special point of view about the quality. Most of the workers have the awareness about the cost of quality, and they have been trying to control the quality of different parts or products in the factory from the beginning to the end of the process.

(3) In production groups, the most important weight has been allocated to cost of sales lost for part $i\left(\Pi i_{i t}\right)$ (TOPSIS: 0.098, FTOPSIS: 0.963), and the lowest important weight is the holding cost of product $j\left(h_{j t p}\right)$ (TOPSIS: 0.015 , FTOPSIS: 0.244$)$. Since the product $j$ is totally dependent on material $i$ and if there is shortages of material in production line, it does not only cause delays in production process, but also imposes loss of costs of sales to the organization. Consequently, the company will not be able to satisfy its customers' demands on time, and in the long run, the company may lose its reputation.

(4) In distribution groups, the most important weight has been allocated to the deficit cost of end period inventory of product $j$ at warehouse $\left(\mathrm{Spp}_{j p t}\right)$ (TOPSIS: 0.253 , FTOPSIS: 0.73 ), and the lowest important weight is the safety stock cost of product $j$ at warehouse $p$ in period $t\left(\right.$ SSP $\left._{j p t}\right)$, (TOPSIS: 0.039 , FTOPSIS: 0.4). As the final product of the factory, the safety stock of product $j$ inventory must be determined; otherwise, there would be shortage for the company.

As human decision-making process usually contains fuzziness and vagueness, FMCDM was adopted to solve the problem. According to the closeness coefficient, we can determine not only the ranking, but also the assessment status of all possible decision makers. In fact, TOPSIS method is very flexible. The method can deal with the ratings of both quantitative and qualitative criteria. It appears from the foregoing sections that TOPSIS method may be a useful additional tool for the problem. The systematic framework for prioritizing and evaluating cost-effectiveness criteria in a fuzzy environment presented in this paper can be easily extended to the analysis of other management decision problems. Other multiattribute evaluation methods such as PROMETHEE, ELECTRE, DEA, and VIKOR in fuzzy environment can also be used. For future research, the authors would like to
TABLE 12: Comparison table from different methods.

\begin{tabular}{ccc}
\hline Criteria and subcriteria & FAHP-TOPSIS & FTOPSIS \\
\hline Supply & $(\mathbf{0 . 7 3 )}$ & $\mathbf{( 0 . 5 3 )}$ \\
$f$ coq $_{Y_{i s}}$ & $(0.209)$ & $(0.87)$ \\
$c_{i t}$ & $(0.204)$ & $(0.832)$ \\
$h_{i t p}$ & $(0.109)$ & $(0.402)$ \\
SSP $_{i p t}$ & $(0.069)$ & $(0.385)$ \\
$\mathrm{TC}_{i s}$ & $(0.021)$ & $(0.155)$ \\
Production & $\mathbf{( 0 . 2 6 9 )}$ & $\mathbf{( 0 . 1 8 2 )}$ \\
$\prod i_{i t}$ & $(0.098)$ & $(0.963)$ \\
$c_{j t}$ & $(0.095)$ & $(0.832)$ \\
$\prod_{i t}$ & $(0.08)$ & $(0.75)$ \\
$c_{r t}$ & $(0.063)$ & $(0.7)$ \\
$\prod_{j t}$ & $(0.0618)$ & $(0.685)$ \\
$\prod j_{j t}$ & $(0.0612)$ & $(0.66)$ \\
$\prod_{r t}$ & $(0.037)$ & $(0.593)$ \\
$\prod_{r t}$ & $(0.035)$ & $(0.402)$ \\
$h_{r p t}$ & $(0.032)$ & $(0.34)$ \\
$\mathrm{SSP}_{r p t}$ & $(0.032)$ & $(0.285)$ \\
$h_{j t p}$ & $(0.015)$ & $(0.244)$ \\
$\mathrm{Distribution}_{\mathrm{Spp}_{j p t}}$ & $\mathbf{( 0 . 1 5 9 )}$ & $\mathbf{( 0 . 7 )}$ \\
$\mathrm{TC}_{j d}$ & $(0.253)$ & $(0.73)$ \\
$\mathrm{Sp}_{j p t}$ & $(0.215)$ & $(0.66)$ \\
$\mathrm{SSP}_{j p t}$ & $(0.047)$ & $(0.53)$ \\
\hline & $(0.039)$ & $(0.4)$ \\
\hline
\end{tabular}

propose an optimization model for material routing in supply chain management which integrates decisions of different functions into a single optimization model.

\section{Indices}

$t$ : Time period $(t=0,1,2, \ldots, T)$

$i$ : Purchased material $(i=0,1,2, \ldots, I)$

$r$ : Part manufactured in workshops $(r=0,1,2, \ldots, R)$

$d$ : Agents for sale $(d=0,1,2, \ldots, D)$

$S$ : Supplier

$j$ : Product manufactured in assembly workshop

$P$ : Warehouse

$e$ : Work stations.

\section{Cost Criteria}

$c_{j t}$ : Cost to produce a unit of product $j$ in period $t$

$c_{i t}$ : Cost to purchase a unit of part $i$ in period $t$

$c_{r t}$ : Cost to produce a unit of part $r$ in period of $t$

$h_{i t p}$ : Cost to hold a unit of part $i$ in period $t$

$h_{j t p}$ : Cost to hold a unit of product $j$ in period $t$

$h_{r t p}$ : Cost to hold a unit of part $r$ in period $t$ 
$\Pi_{j t}$ : $\quad$ Unit cost of deficit for product $j$ in period $t$

$\Pi j_{j t}$ : Unit cost of sales lost for product $j$ in period $t$

$\Pi_{i t}$ : $\quad$ Unit cost of deficit for part $i$ in period $t$

$\Pi i_{i t}$ : Unit cost of sales lost for part $i$ in period $t$

$\Pi_{r t}$ : Unit cost of deficit for part $r$ in period $t$

$\Pi r_{r t}$ : Unit cost of sales lost for part $r$ in period $t$

$\mathrm{Sp}_{j p t}$ : Storage cost of end of period inventory of product $j$ at warehouse $p$ in period $t$

$\mathrm{Spp}_{j p t}$ : Deficit cost of end of period inventory of product $j$ at warehouse $p$ in period $t$

$\mathrm{TC}_{j d}$ : Transportation cost of one unit of product $j$ to distributor $d$

$\mathrm{TC}_{i s}$ : Transportation cost of one unit of part $i$ from supplier $s$

$\mathrm{SSP}_{j p t}$ : Safety stock cost of product $j$ at warehouse $p$ in period $t$

$\mathrm{SSP}_{i p t}$ : Safety stock cost of part $i$ at warehouse $p$ in period $t$

$\mathrm{SSP}_{r p t}$ : Safety stock cost of part $r$ at warehouse $p$ in period $t$

$f \operatorname{coq}_{Y_{i s}}$ : Total cost of quality (including prevention and appraisal costs) for supplier $s$ per good part $i$ as a function of $Y_{i}$, the level of proportion of nonquality components. This is equivalent to the total cost of quality.

\section{Acknowledgment}

The author would like to deeply thank the anonymous referees for their valuable comments.

\section{References}

[1] M. Christopher, Logistics and Supply Chain Management, Pearson Education, Harlow, UK, 1998.

[2] S. Li, B. Ragu-Nathan, S. Subba Rao, and T. S. Ragu-Nathan, "The impact of supply chain management practices on competitive advantage and organizational performance," Omega, vol. 34, no. 2, pp. 107-124, 2006.

[3] W. A. Jauhari, "Integrated inventory model for three-layer supply chains with stochastic demand," International Journal of Operational Research, vol. 13, no. 3, pp. 295-317, 2012.

[4] H. Deng, C. H. Yeh, and R. J. Willis, "Inter-company comparison using modified TOPSIS with objective weights," Computers and Operations Research, vol. 27, no. 10, pp. 963-973, 2000.

[5] D. L. Olson, "Comparison of weights in TOPSIS models," Mathematical and Computer Modelling, vol. 40, no. 7-8, pp. 721727, 2004.

[6] E. H. Sabri and B. M. Beamon, "A multi-objective approach to simultaneous strategic and operational planning in supply chain design," Omega, vol. 28, no. 5, pp. 581-598, 2000.

[7] M. A. Cohen and H. L. Lee, "Resource development analysis of global manufacturing and distribution networks," Journal of Manufacturing and Operation Management, vol. 2, pp. 81-104, 1989.

[8] D. Petrovic, R. Roy, and R. Petrovic, "Modelling and simulation of a supply chain in an uncertain environment," European Journal of Operational Research, vol. 109, no. 2, pp. 299-309, 1998.
[9] H. Pirkul and V. Jayaraman, "A multi-commodity, multi-plant, capacitated facility location problem: formulation and efficient heuristic solution," Computers and Operations Research, vol. 25, no. 10, pp. 869-878, 1998.

[10] C. J. Vidal and M. Goetschalck, "Modeling the effect of uncertainties on global logistics systems," Journal of Business Logistics, vol. 21, no. 1, pp. 95-121, 2000.

[11] Y. H. Lee and S. H. Kim, "Production-distribution planning in supply chain considering capacity constraints," Computers and Industrial Engineering, vol. 43, no. 1-2, pp. 169-190, 2002.

[12] H. Yin, J. Zheng, and X. Wang, "Multi-agent based supply chain modeling and bidding," in Proceedings of the 5th World Congress on Intelligent Control and Automation (WCICA '04), pp. 31873191, Hangzhou, China, June 2004.

[13] F. Nonino and R. Panizzolo, "Integrated production/distribution planning in the supply chain: the Febal case study," Supply Chain Management, vol. 12, no. 2, pp. 150-163, 2007.

[14] A. Ross and V. Jayaraman, "An evaluation of new heuristics for the location of cross-docks distribution centers in supply chain network design," Computers and Industrial Engineering, vol. 55, no. 1, pp. 64-79, 2008.

[15] V. Jayaraman and A. Ross, "A simulated annealing methodology to distribution network design and management," European Journal of Operational Research, vol. 144, no. 3, pp. 629-645, 2003.

[16] B. J. Jeong, H. S. Jung, and N. K. Park, "A computerized causal forecasting system using genetic algorithms in supply chain management," Journal of Systems and Software, vol. 60, no. 3, pp. 223-237, 2002.

[17] Y. Yu, H. Chen, and F. Chu, "A new model and hybrid approach for large scale inventory routing problems," European Journal of Operational Research, vol. 189, no. 3, pp. 1022-1040, 2008.

[18] M. E. Seliaman, "Using complete squares method to optimize replenishment policies in a four-stage supply chain with planned backorders," Advances in Decision Sciences, vol. 2011, Article ID 745896, 9 pages, 2011.

[19] A. Amirteimoori and L. Khoshandam, "A data envelopment analysis approach to supply chain efficiency," Advances in Decision Sciences, vol. 2011, Article ID 608324, 8 pages, 2011.

[20] C. L. Hwang and K. Yoon, Multiple Attribute Decision Making Methods and Application, Springer, New York, NY, USA, 1981.

[21] C. T. Chen, "Extensions of the TOPSIS for group decisionmaking under fuzzy environment," Fuzzy Sets and Systems, vol. 114, no. 1, pp. 1-9, 2000.

[22] Y. M. Wang and T. M. S. Elhag, "Fuzzy TOPSIS method based on alpha level sets with an application to bridge risk assessment," Expert Systems with Applications, vol. 31, no. 2, pp. 309-319, 2006.

[23] T. C. Wang and T. H. Chang, "Application of TOPSIS in evaluating initial training aircraft under a fuzzy environment," Expert Systems with Applications, vol. 33, no. 4, pp. 870-880, 2007.

[24] T. Yang and C. C. Hung, "Multiple-attribute decision making methods for plant layout design problem," Robotics and Computer-Integrated Manufacturing, vol. 23, no. 1, pp. 126-137, 2007.

[25] T. C. Wang and H. D. Lee, "Developing a fuzzy TOPSIS approach based on subjective weights and objective weights," Expert Systems with Applications, vol. 36, no. 5, pp. 8980-8985, 2009. 
[26] C. Kahraman, G. Büyüközkan, and N. Y. Ateş, "A two phase multi-attribute decision-making approach for new product introduction," Information Sciences, vol. 177, no. 7, pp. 1567-1582, 2007.

[27] M. Dağdeviren, S. Yavuz, and N. Kilinç, "Weapon selection using the AHP and TOPSIS methods under fuzzy environment," Expert Systems with Applications, vol. 36, no. 4, pp. 81438151, 2009.

[28] A. T. Gumus, "Evaluation of hazardous waste transportation firms by using a two step fuzzy-AHP and TOPSIS methodology," Expert Systems with Applications, vol. 36, no. 2, pp. 40674074, 2009.

[29] C. C. Sun and G. T. R. Lin, "Using fuzzy TOPSIS method for evaluating the competitive advantages of shopping websites," Expert Systems with Applications, vol. 36, no. 9, pp. 11764-11771, 2009.

[30] F. Torfi and A. Rashidi, "Selection of project managers in construction Firms using analytic hierarchy process (AHP) and fuzzy Topsis: a case study," Journal of Construction in Developing Countries, vol. 16, no. 1, pp. 69-89, 2011.

[31] E. Armero, M. S. García-Cascales, M. D. Gómez-López, and M. T. Lamata, "Decision making in uncertain rural scenarios by means of fuzzy TOPSIS method," Advances in Decision Sciences, vol. 2011, Article ID 937092, 15 pages, 2011.

[32] S. J. Chen and C. L. Hwang, Fuzzy Multiple Attribute Decision Making, vol. 375 of Lecture Notes in Economics and Mathematical System Series, Springer, New York, NY, USA, 1992.

[33] G. Svensson, "Holistic and cross-disciplinary deficiencies in the theory generation of supply chain management," Supply Chain Management, vol. 8, no. 4, pp. 303-316, 2003.

[34] C. Y. Wong, J. S. Arlbjørn, and J. Johansen, "Supply chain management practices in toy supply chains," Supply Chain Management, vol. 10, no. 5, pp. 367-378, 2005.

[35] M. E. Johnson, "Learning from toys: lessons in managing supply chain risk from the toy industry," California Management Review, vol. 43, no. 3, pp. 106-124, 2001.

[36] M. Christopher, R. Lowson, and H. Peck, "Creating agile supply chains in the fashion industry," International Journal of Retail \& Distribution Management, vol. 32, no. 8, pp. 367-376, 2004.

[37] I. Ertuğrul and N. Karakaşoğlu, "Performance evaluation of Turkish cement firms with fuzzy analytic hierarchy process and TOPSIS methods," Expert Systems with Applications, vol. 36, no. 1, pp. 702-715, 2009. 


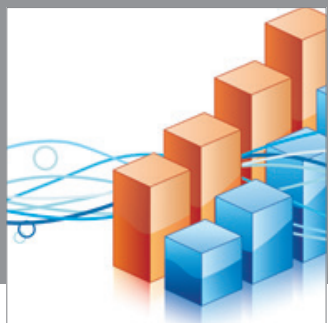

Advances in

Operations Research

mansans

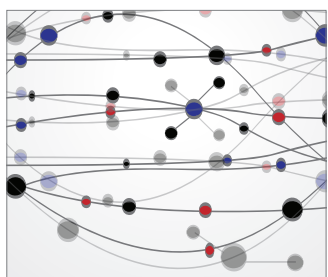

The Scientific World Journal
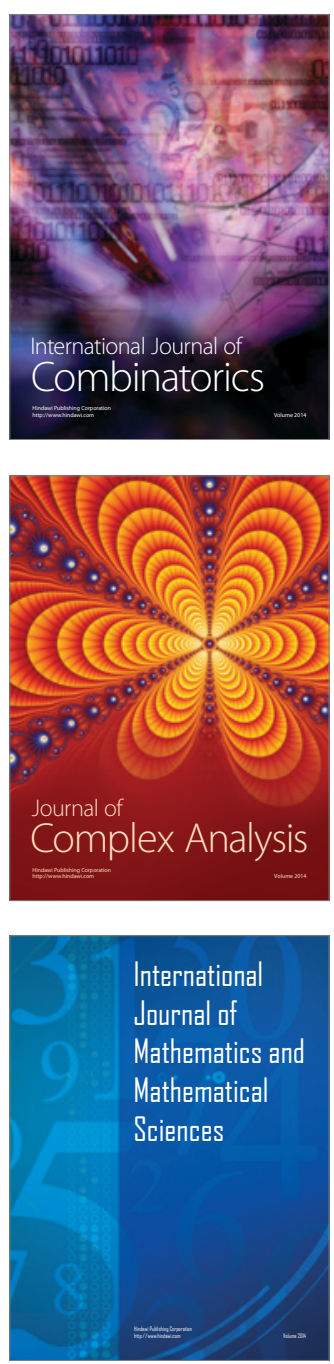
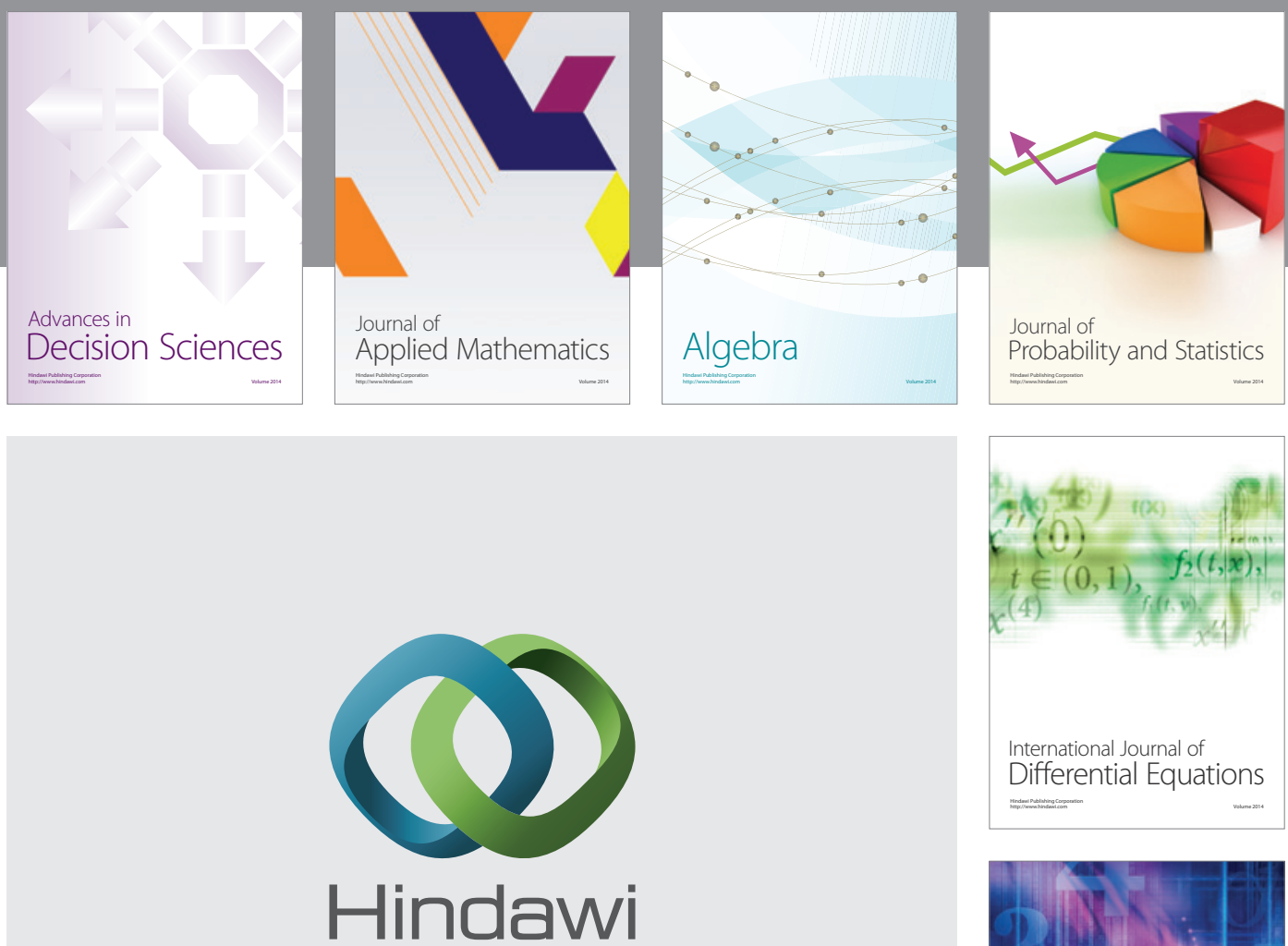

Submit your manuscripts at http://www.hindawi.com
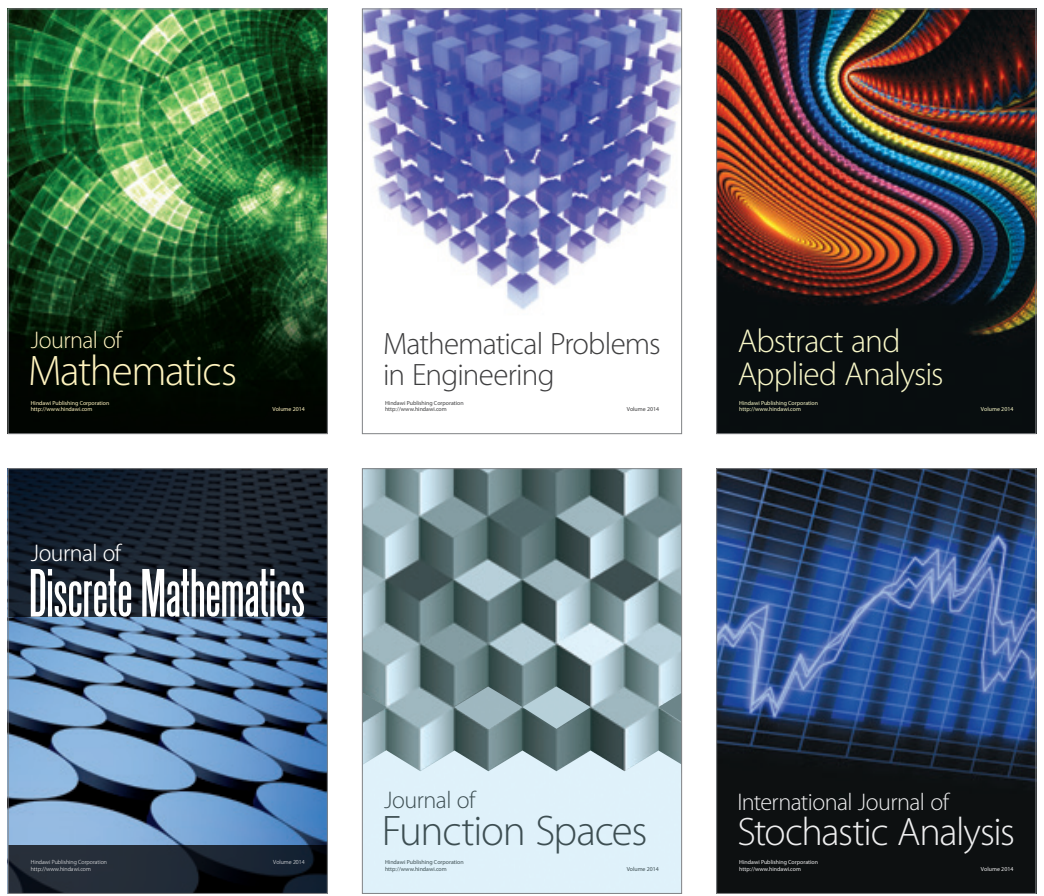

Journal of

Function Spaces

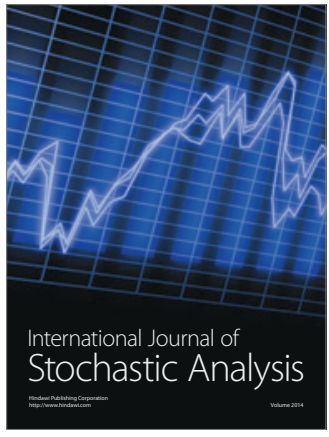

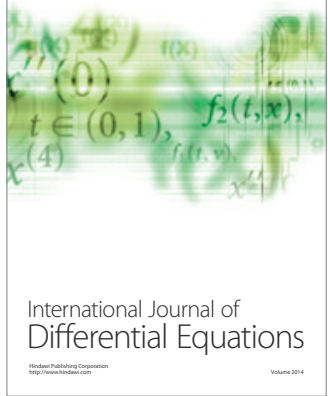
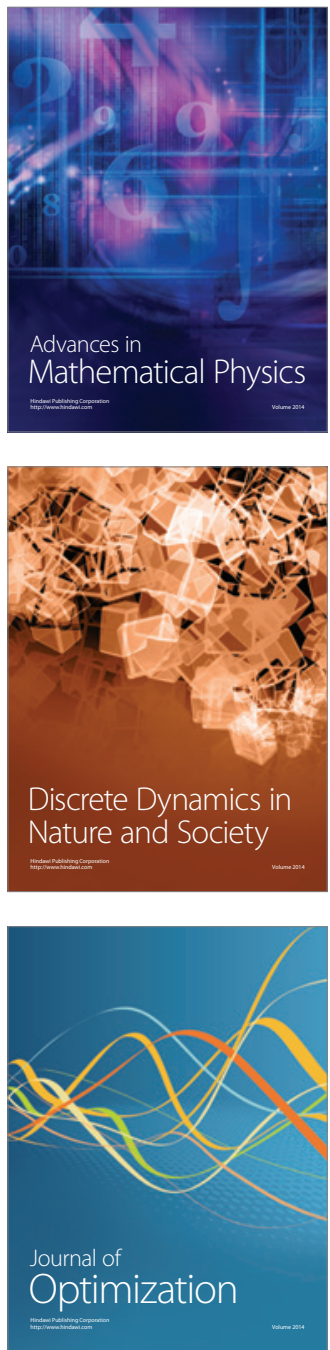